H. Umemura

Nagoya Math. J.

Vol. 87 (1982), 59-78

\title{
MAXIMAL ALGEBRAIC SUBGROUPS OF THE CREMONA GROUP OF THREE VARIABLES \\ IMPRIMITIVE ALGEBRAIC SUBGROUPS OF EXCEPTIONAL TYPE
}

\author{
HIROSHI UMEMURA
}

Enriques and Fano [4], [5] classified all the maximal connected algebraic subgroups of $\mathrm{Cr}_{3}$. Our aim is to give modern and rigorous proofs to their results. In [10], we studied the primitive subgroups. In this paper, we deal with exceptional imprimitive groups. The imprimitivity is an analytic notion. The natural translation of the imprimitivity in algebraic geometry is the de Jonquières type operation (definition (2.1)). Every de Jonquières type operation is imprimitive. However, the difference of these notions is subtle. The imprimitive algebraic operations in $\mathrm{Cr}_{3}$ which are not of de Jonquières type are rather exceptional; there are only 3 such operations (theorem (3.26)). This paper together with [10] recovers all the results on $\mathrm{Cr}_{3}$ of Enriques and Fano [4]. It remains only to reconstruct Fano's classification [5] of the de Jonquières type operations, which shall be done in our forthcoming paper.

Our technique is rather old; the classification of 3 dimensional primitive operations, a very easy part of invariant theory which are of 19th century, combined with the theory of algebraic groups and transformation spaces of A. Weil. As the 4 dimensional primitive law chunks of analytic operations are classified, our method can be applied to the 4 dimensional Cremona group $\mathrm{Cr}_{4}$. We use the notations and the conventions of [10]. Therefore all manifolds, analytic groups, algebraic varieties, etc. are defined over $C$. The transformation spaces $X$ of analytic or algebraic law chunk or operation $(G, X)$ are connected. However, a differences lies in the language that we employ. Here is our French-English dictionary:

Received February 25, 1980. 


\author{
French \\ groupuscule \\ morceau de loi d'opération
}

\author{
English \\ group germ \\ law chunk of operation
}

\section{§ 1. Analytic preliminaries}

We supply $\S 1$ of [10]. We recall known facts and nothing is new in this section. We work in the category of the complex manifolds. All manifolds, analytic groups, etc. are analytic over $C$. For the definitions of analytic group germ and law chunk of analytic operation, we refer to [10].

Definition 1.1. Let $\mathfrak{g}$ be an Lie algebra and $X$ a (connected) manifold. A homomorphism $\alpha$ of Lie algebra $g$ to the Lie algebra $H^{\circ}\left(X, T_{X}\right)$ of the holomorphic vector fields on $X$ is called an infinitesimal operation of $\mathfrak{g}$ on $X$. An infinitesimal operation is denoted by $(\mathfrak{g}, X)$ without making $\alpha$ precise. Let $\left(\mathfrak{g}^{\prime}, X^{\prime}\right)$ be another infinitesimal operation defined by $\alpha^{\prime}: \mathfrak{g}^{\prime} \rightarrow$ $H^{0}\left(X^{\prime}, T_{X^{\prime}}\right)$. A morphism $(\varphi, f)$ of infinitesimal operations of $(\mathfrak{g}, X)$ to $\left(\mathfrak{g}^{\prime}, X^{\prime}\right)$ consists of a morphism of Lie algebras $\varphi: \mathfrak{g} \rightarrow \mathfrak{g}^{\prime}$ and a morphism $f$ of a non-empty open subset of $X$ to $X^{\prime}$ such that $f_{*} \alpha(D)=\alpha^{\prime}(\varphi(D))$.

Let $G$ be a analytic group germ, $g$ its Lie algebra and $X$ a manifold. Let $(G, X)$ be an law chunk of analytic operation. As we have seen in [10], we can associate to $(G, X)$ an infinitesimal operation of $g$ on $X$. We have a converse (corollaire $1, \mathrm{Ch} . \mathrm{III}, \S 5, \mathrm{n}^{\circ} 7$, Bourbaki [2]).

Proposition 1.2. Suppose that $X$ is paracompact. Let $\alpha: \mathfrak{g} \rightarrow H^{0}\left(X, T_{X}\right)$ be an infinitesimal operation of $\mathrm{g}$ on $X$. Then, there exists a unique law chunk of analytic operation $(G, X)$ such that the infinitesimal operation associated to $(G, X)$ is $\alpha$.

When we consider law chunks, we are in local questions. Hence the hypothesis that $X$ is paracompact is not a serious condition. Proposition 1.2 and the following proposition show that to consider law chunk is equivalent to consider infinitesimal operations.

Proposition 1.3. Let $(G, X),\left(G^{\prime}, X^{\prime}\right)$ be law chunks of analytic operation and $(\mathfrak{g}, X),\left(\mathfrak{g}^{\prime}, X^{\prime}\right)$ their infinitesimal operations. We have $\mathrm{Hom}_{\text {law chunks }}$ $\left((G, X),\left(G^{\prime}, X^{\prime}\right)\right) \simeq \operatorname{Hom}_{\text {infinttesimal operations }}\left((\mathfrak{g}, X),\left(\mathfrak{g}, X^{\prime}\right)\right)$.

See proposition 11, Ch. III, $\S 4, n^{\circ} 7$, Bourbaki [2].

Let $\alpha: \mathfrak{g} \rightarrow H^{0}\left(X, T_{X}\right)$ be an infinitesimal operation. Let $V$ be the sub- 
set of $X$ consisting of $x \in X$ such that $\operatorname{dim} \alpha(\mathrm{g})_{x}$ is maximal. Then $V$ is an open set and $X-V$ is an analytic set.

Definition 1.4. Let $(g, X)$ be an infinitesimal operation. We say that $(\mathfrak{g}, X)$ is generically transitive if there exists a point $x \in X$ such that $\alpha(\mathfrak{g})_{x}=$ $T_{x}$. The infinitesimal operation $(\mathfrak{g}, X)$ is said to be generically intransitive if $(g, X)$ is not generically transitive. A law chunk of analytic operation is generically transitive (resp. intransitive) by definition if its associated infinitesimal operation is generically transitive (resp. intransitive). For the definition of primitive (resp. imprimitive) law chunks we refer to $\S 1$ in [10].

Proposition 1.5. If $(G, X)$ is generically intransitive and $\operatorname{dim} X \geq 2$, $(G, X)$ is imprimitive.

Proof. The morphism $\alpha: \mathfrak{g} \rightarrow H^{0}\left(X, T_{X}\right)$ defines a system of vector fields. On a non-empty open subset $U$ of $X, \alpha(\mathfrak{g}) \mid U$ is a differential system satisfying the integrability condition. Hence by the Frobenius theorem, there exists locally the quotient $W$ of $X$ by the operation of $G$. Therefore, we have a morphism of infinitesimal operations $(\mathfrak{g}, X) \rightarrow(0, W)$ and the $\operatorname{dim} W$ is equal to $d=\operatorname{dim} X-\operatorname{dim} \alpha(\mathfrak{g})(x) x \in U$. Hence, we get a morphism of law chunks of analytic operation $(G, X) \rightarrow(E, W)$ where $E$ is the unit group by proposition 1.3. This shows $(G, X)$ is imprimitive.

Now we study the generically transitive case. Let $G$ be an analytic group and $\mathfrak{g}$ its Lie algebra. Let $H$ be a connected analytic subgroup of $G$. The subgroup $H$ is not necessarily closed and the quotient space $G / H$ does not always exist. But it exists locally by the Frobenius theorem. In fact, $H$ operates on $G$ from the right and this operation defines on $G$ a differential system satisfying the integrability condition. Hence by the Frobenius theorem the quotient space exists locally around the unit element $e$. We denote this local quotient space by $(G / H)^{\prime}$. The operation from the left of $G$ on $G$ itself leaves this differential system invariant. Hence, $G$ operates locally on $(G / H)^{\prime}$. We get a law chunk of analytic operation $\left(G,(G / H)^{\prime}\right)$. The law chunk of analytic operation $\left(G,(G / H)^{\prime}\right)$ is uniquely determined up to local isomorphism.

Lemma 1.6. Let $G$ be an analytic group, $X$ a manifold and $(G, X)$ be a generically transitive law chunk of analytic operation. Then there exists an analytic subgroup $H$ of $G$ such that $(G, X)$ is locally isomorphic 
to $\left(G,(G / H)^{\prime}\right)$.

Proof. Take a point $x \in X$ such that $\mathfrak{g}_{x}$ is equal to $T_{x}$. Let $\mathfrak{h}$ be the kernel $\mathrm{g} \rightarrow T_{x}$ and $H$ the connected analytic subgroup of $G$ with Lie algebra $\mathfrak{h}$. Then the mapping $g \mapsto g x$ for $g \in G$ sufficiently close to $e$ defines a local morphism of law chunks of analytic operations of $(G, G)$ to $(G, X)$. This morphism passes to the quotient $\left(G,(G / H)^{\prime}\right)$ and gives local isomorphism of $\left(G,(G / H)^{\prime}\right)$ to $(G, X)$.

Proposition 1.7. Let $G$ be an analytic group with Lie algebra $g$ and $H$ be a connected analytic subgroup. Then the law chunk of analytic operation $\left(G,(G / H)^{\prime}\right)$ is primitive if and only if the Lie algebra $\mathfrak{h}$ of $H$ is maximal, among the Lie subalgebras $\subsetneq \mathrm{g}$.

Proof. If $\mathfrak{h}$ is not maximal, then let $\mathfrak{f}$ be a Lie algebra such that $\mathfrak{h} \subseteq \mathfrak{l} \subseteq \mathfrak{g}$. The argument above shows there exists a morphism of law chunks of analytic operation $\left(G,(G / H)^{\prime}\right) \rightarrow\left(G,(G / K)^{\prime}\right)$ where $K$ is the

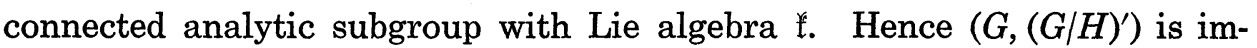
primitive. Conversely, if $\left(G,(G / H)^{\prime}\right)$ is imprimitive, let $(\mathrm{Id}, f):\left(G,(G / H)^{\prime}\right)$ $\rightarrow(G, X)$ be a morphism of imprimitivity. Considering the image of $f$, we may assume that $f$ is a submersion hence $(G, X)$ is generically transitive. Therefore, we may assume, by lemma 1.6 , that $(G, X)=\left(G,(G / K)^{\prime}\right)$ for a connected analytic subgroup $K \subset G$. Furthermore, we may assume that $f$ is defined at $\bar{e} \in(G / H)^{\prime}$ and is the natural projection $(G / H)^{\prime} \rightarrow(G / K)^{\prime}$ so that the image $f(\bar{e})$ of $\bar{e} \in(G / H)^{\prime}$ coincides with $\bar{e} \in(G / K)^{\prime}$.

Proposition 1.8. Let $G$ be an analytic group and $H$ be a closed analytic subgroup of $G$. Let $\mathfrak{g}, \mathfrak{h}$ be the Lie algebra of the subgroup $G$ and $H$ respectively. Then the following conditions are equivalent:

(1) The analytic operation $(G, G / H)$ is primitive

(2) There exists no Lie subalgebra $\mathfrak{f}$ of $\mathrm{g}$ such that $\mathfrak{h} \sqsubseteq \mathfrak{g}$.

Proof. If we notice that $(G, G / H)$ is (locally) isomorphic to $\left(G, G / H^{0}\right)$ as a law chunk of analytic operation, the proposition follows from proposition (1.7).

\section{§2. Algebraic preliminaries}

In this section, we work in the category of algebraic varieties. When we speak of open subsets, they are open evidently in Zariski topology. Everything works analogously as in the analytic case. The algebraic 
counterpart of imprimitivity is de Jonquières type transformation.

Definition 2.1. Let $G$ be an algebraic group germ and $(G, X)$ a law chunk of algebraic operation. We say that $(G, X)$ is of de Jonquières type if there exist a law chunk of algebraic operation $\left(G^{\prime}, X^{\prime}\right)$, a morphism of law chunks of algebraic operation $(\varphi, f):(G, X) \rightarrow\left(G^{\prime}, X^{\prime}\right)$ and a (Zariski) open subset $U$ of $X$ such that $f$ is regular on $U$ and $0<\operatorname{dim} f(U)<\operatorname{dim} X$.

Proposition 2.2. Let $(G, X)$ be an algebraic operation. The following conditions are equivalent:

(1) $(G, X)$ is of de Jonquières type.

(2) There exist an algebraic operation $\left(G^{\prime}, X^{\prime}\right)$, a morphism of law chunks of algebraic operation $(\varphi, f):(G, X) \rightarrow\left(G^{\prime}, X^{\prime}\right)$ and a (Zariski) open subset $U$ of $X$ such that $f$ is regular on $U$ and $0<\operatorname{dim} f(U)<\operatorname{dim} X$.

(3) There exist an algebraic operation $\left(G^{\prime}, X^{\prime}\right)$ with $G^{\prime}=G$, a morphism of law chunks of algebraic operation $(\varphi, f):(G, X) \rightarrow\left(G^{\prime}, X^{\prime}\right)$ and a (Zariski) open subset $U$ of $X$ satisfying the same condition as in (2).

Proof. The implications $(3) \Rightarrow(2) \Rightarrow(1)$ is trivial. The assertion (2) follows from (1) by theorem 1, Rosenlicht [8]. To obtain (3) from (2), it suffices to replace $G^{\prime}$ by the image $\varphi(G)$.

If a law chunk of algebraic operation $(G, X)$ is of de Jonquières type, then its associated law chunk of analytic operation $\left(G^{a n}, X^{a n}\right)$ is imprimitive. As we shall see later there are law chunks of algebraic operations $(G, X)$ which are imprimitive but not of de Jonquières type. But when $X$ is rational and of dimension 3, most of imprimitive law chunks of algebraic operation are of de Jonquières type.

Theorem 2.3 (Rosenlicht [8]). Let $G$ be an algebraic group and $(G, X)$ an algebraic operation. Then there exist an algebraic operation $\left(G, X^{\prime}\right)$ and a morphism $\left(\operatorname{Id}_{G}, f\right):(G, X) \rightarrow\left(G, X^{\prime}\right)$ of law chunks of algebraic operation and open set $U \subset X$ such that $f$ is generically surjective, $f$ is regular on $U$ and such that, for any two points $x, y \in U$, the image $f(x)$ coincides with $f(y)$ if and only if $x$ and $y$ lie in an orbit of $G$.

The variety $X^{\prime}$ is called the variety of $G$-orbits on $X$.

Definition 2.4. We say that an algebraic operation $(G, X)$ is generically intransitive if there does not exist an open orbit on $X$.

COROLlary 2.5. Every generically intransitive algebraic operation on 
an algebraic variety of dimension $\geq 2$ is of de Jonquières type.

CoRollary 2.6. Every algebraic operation of solvable linear algebraic groups on an algebraic variety of dimension $\geq 2$ is of de Jonquières type.

Let $G$ be a solvable algebraic group, $X$ an algebraic variety of dimension $\geq 2$ and $(G, X)$ be an algebraic operation. We may assume that the map $G \rightarrow$ Aut $X$ defined by the operation is injective. Since $G$ is solvable, there exists a normal subgroup $H$ of dimension 1 of $G$. Since the dimension of $X \geq 2$, the operation $(H, X)$ is generically intransitive. Since $H$ is normal, $G$ interchanges the orbits of $H$ on $X$. Hence, if $X^{\prime}$ is the variety of $H$-orbits on $X, G$ acts rationally on $X^{\prime}$ and we have natural morphism $\left(\operatorname{Id}_{G}, f\right):(G, X) \rightarrow\left(G, X^{\prime}\right)$ of law chunks of algebraic operation.

The following proposition shows that we can globalize a local algebraic morphism of homogeneous spaces.

Proposition 2.7 (Rosenlicht [8]). Let $G_{1}, G_{2}$ be algebraic groups and $H_{1}, H_{2}$ be closed algebraic subgroups of $G_{1}, G_{2}$. Let $(\varphi, f):\left(G_{1}, G_{1} / H_{1}\right) \rightarrow\left(G_{2}\right.$, $\mathrm{G}_{2} / \mathrm{H}_{2}$ ) be a morphism of law chunks of algebraic operation. Then the morphism $\varphi$ and $f$ are regular hence $(\varphi, f)$ is a morphism of algebraic operations.

Corollary 2.8. Let $G$ be an algebraic group. Let $H$ be a closed algebraic subgroup. The algebraic operation $(G, G / H)$ is of de Jonquières type if and only if exists a closed algebraic subgroup $K$ such that $H \subset K$ and $\operatorname{dim} H<\operatorname{dim} K<\operatorname{dim} G$.

Proof of the Corollary. If $(G, G / H)$ is of de Jonquières type, there exists an algebraic operation $\left(G, X^{\prime}\right)$ and a morphism of law chunks of algebraic operation $(\varphi, f):(G, G / H) \rightarrow\left(G, X^{\prime}\right)$ satisfying the conditions of proposition 2.2. Since $(G, G / H)$ is homogeneous, we may assume $\left(G, X^{\prime}\right)$ is a homogeneous space $(G, G / K)$. Then, by proposition $2.6,(\varphi, f)$ is a morphism of algebraic operations. Furthermore, we may assume the image $f(H)$ of the coset $H \in G / H$ is $K \in G / K$. The subgroup $K$ satisfies our requirement. Conversely, if we have a closed algebraic subgroup $K \subset G$ such that $H \subset K$ and $\operatorname{dim} H<\operatorname{dim} K<\operatorname{dim} G$, the canonical map $(G, G / H)$ $\rightarrow(G, G / K)$ satisfies the condition of definition 2.1 .

Remark 2.9. So far, it seems to us that everything works analogously in analytic category and algebraic category: We have a correspondence; imprimitive $\longleftrightarrow$ de Jonquières type 
proposition $1.5 \longleftrightarrow$ corollary 2.5

proposition $1.7 \longleftrightarrow$ corollary 2.8 .

But a big difference is that by Rosenlicht [8], a law chunk of algebraic operation can be globalized but we can not always globalize a law chunk of analytic operation. For example, there exist a simply connected analytic group $G$ with Lie algebra $g$ and Lie subalgebra $\mathfrak{h}$ such that the analytic subgroup corresponding to $\mathfrak{h}$ is not closed.

\section{§3. Algebraic subgroups in $\mathbf{C r}_{3}$}

The Cremona group $\mathrm{Cr}_{n}$ of $n$-variables is, by definition, the group of all the birational automorphisms of the projective space $\boldsymbol{P}_{n}$. The group $\mathrm{Cr}_{n}$ is isomorphic to the group of all the $\boldsymbol{C}$-automorphisms of the rational function field $C\left(X_{1}, \cdots, X_{n}\right)$ of $n$-variables. To give a connected algebraic subgroup of the Cremona group $\mathrm{Cr}_{n}$ is equivalent to give a connected algebraic group $G$, an algebraic variety $X$, an algebraic operation $(G, X)$, a birational map $f: X \cdots \rightarrow P_{n}$ such that

(3.1) the map $G \rightarrow$ Aut $X$ induced by the operation $(G, X)$ is injective (See Umemura [10], theorem 1 Rosenlicht [8]). If we replace the birational map $f$ by another birational map $f^{\prime}$ of $X$ to $\boldsymbol{P}_{n}$, we get another algebraic subgroup $G^{\prime}$ of $\mathrm{Cr}_{n}$ conjugate to $G$. All the algebraic subgroups of $\mathrm{Cr}_{n}$ conjugate to $G$ is obtained in this manner. Hence, there is a $1-1$ correspondence between the conjugacy classes of the connected algebraic subgroup of $\mathrm{Cr}_{n}$ and the local isomorphism classes of the algebraic operations $(G, X)$ satisfying the condition (3.1) such that $G$ is connected and $X$ is rational and $n$ dimensional. As we are interested in the conjugacy classes, we shall consider the algebraic operations. The following theorem of Matsumura-Nishi estimates the dimension of the abelian part of an algebraic group operating on a variety.

Theorem 3.2 (Matsumura [7]). Let $G$ be an algebraic group, $(G, X)$ an algebraic operation satisfying the condition (3.1) and $G \rightarrow A$ a surjective homomorphism of $G$ to an abelian variety $A$. Then, the dimension of $A$ $\leq$ the irregularity of $X(=$ the dimension of the Albanese variety of a nonsingular projective model of $X$ ).

When we treat rational varieties $X$, the irregularity is 0 . It follows, from the structure theorem of algebraic groups; 
CoROLlary 3.3. Let $(G, X)$ be an algebraic operation satisfying the condition (3.1). If $X$ is rational, the algebraic group $G$ is linear.

Proposition 3.4. Let $G$ be a connected algebraic subgroup of $\mathbf{C r}_{n}$. The following are equivalent.

(1) There exists a primitive algebraic operation $(G, X)$ realizing the subgroup $G \subset \mathrm{Cr}_{n}$.

(2) Every algebraic operation realizing the subgroup $G \subset \mathrm{Cr}_{n}$ is primitive.

Proof. It is sufficient to show (2) is a consequence of (1). Let $\left(G, X^{\prime}\right)$ be an algebraic operation realizing the subgroup $G \subset \mathrm{Cr}_{n}$. By corollary p. 404 Rosenlicht [8], there exist open subsets $U, U^{\prime}$ of $X$ and $X^{\prime}$ which are invariant and a isomorphism of algebraic operations $(G, U) \rightarrow\left(G, U^{\prime}\right)$. If $\left(G^{a n}, X^{\prime a n}\right)$ were imprimitive, $\left(G^{a n}, U^{\prime a n}\right)$ would be imprimitive since the Zariski open subset $U^{\prime}$ is dense in the usual topology. Consequently ( $G^{a n}$, $U^{a n}$ ) would be imprimitive.

CoRollary 3.5. Let $G$ be a connected algebraic subgroup of $\mathrm{Cr}_{n}$. The following are equivalent:

(1) There exists an imprimitive algebraic operation $(G, X)$ realizing the subgroup $G \subset \mathrm{Cr}_{n}$.

(2) Every algebraic operation realizing the subgroup $G \subset \mathrm{Cr}_{n}$ is imprimitive.

Definition 3.6. Let $G$ be a connected algebraic subgroup of $\mathrm{Cr}_{n}$. The subgroup is said to be primitive (resp. imprimitive) if it satisfies the conditions of proposition (3.4) (resp. corollary (3.5)).

TheOREm 3.7. Let $G$ be a connected algebraic group, $X$ a rational variety of dimension 3 and $(G, X)$ an algebraic operation satisfying the condition (3.1). If $(G, X)$ is imprimitive and the dimension of $G \geq 4$, then $(G, X)$ is of de Jonquières type.

Proof. By proposition 1.2, corollary 3.3 corollary 2.5 and corollary 2.6 we may assume that $G$ is linear, $(G, X)$ has an open orbit and $G$ is not solvable. Then, the algebraic operation $(G, X)$ is birationally isomorphic to a homogeneous space $(G, G / H)$, hence we can assume $(G, X)$ is a homogeneous space $(G, G / H)$. It is sufficient to show by Corollary 2.8 that there exists an algebraic subgroup $H^{\prime}$ such that (a) $H \sqsubseteq H^{\prime} \subsetneq G, \operatorname{dim} H$ $<\operatorname{dim} H^{\prime}$. 
Lemma 3.8. Let $N \neq\{e\}$ be a normal subgroup of $G$. Then, the subgroup $N$ is not contained in $H$.

Proof of the lemma. In fact if $N \subset H$, for $g \in G$ we have $N g H=g N H$ $=g H$. The subgroup $N$ operates trivially on $G / H$, which contradicts the hypothesis that $(G, G / H)$ satisfies the condition 3.1 .

It follows from the lemma that if $N \neq\{e\}$ is a closed connected normal algebraic subgroup of $G$, then $H N$ is a closed algebraic subgroup of $G$ such that $H \subset H N, \operatorname{dim} H<\operatorname{dim} H N$, since the connected component $(H N)^{0}=H^{0} N^{0}=H^{0} N$. Hence moreover if $H N \neq G$, the subgroup $H N$ satisfies the condition of corollary 2.8. Therefore, we may (and do) assume that, (3.9) for any connected normal algebraic subgroup $N \neq\{e\}$, we have $N H=G$. We study two cases separately.

Case (a). The group $G$ is not semi-simple.

Let $R$ be the radical of $G$ and $R_{u}$ the unipotent part of $R$. The subgroups $R$ and $R_{u}$ are connected and normal in $G$.

Subcase $(\mathrm{a}-1) . \quad R_{u} \neq\{e\}$.

Let $Z$ be the center of $R_{u}$. Since $R_{u} \neq\{e\}$, the dimension of $Z$ is positive. Let $Z^{0}$ be the connected component of $Z$. Since $R_{u}$ is normal, the center $Z$ is normal in $G$. Therefore $Z$ is normal in $G$. It follows from our hypothesis (3.9) that $Z^{0} H=G$. The group $Z^{0}$ is connected, commutative and unipotent. Hence, the group $Z^{0}$ is isomorphic to $\boldsymbol{G}_{a}^{r}$ for certain positive integer $r$. The equality $Z^{0} H=G$ shows that the commutative algebraic group $Z^{0}$ operates on $G / H$ transitively. The variety $Z^{0} / H \cap Z^{0}$ is isomorphic to $G / H$. Since the operation $\left(Z^{0}, H / H \cap Z^{0}\right)=\left(Z^{0}\right.$, $G / H$ ) satisfies the condition (3.1) and $Z^{0}$ is abelian, the intersection $H \cap Z^{0}$ reduces to $e$. It follows that $G=Z^{0} H$ is the semi-direct product. Let $g$, $\jmath, \mathfrak{h}$ be the Lie algebras of $G, Z, H$ respectively. Then $g=z \oplus \mathfrak{h}$ as a vector space. The imprimitivity of the operation $(G, G / H)$ is equivalent that the Lie subalgebra $\mathfrak{h} \subset \mathrm{g}$ is not maximal by proposition 1.8. Hence there exists a proper Lie subalgebra $\mathfrak{l}$ of $\mathfrak{g}$ such that $\mathfrak{h} \subset \mathfrak{t} \subset \mathfrak{g}$. This is equivalent to say that there exists a proper linear subspace $0 \neq \mathfrak{a} \subsetneq z$ such that $\mathfrak{a}$ is invariant through the adjoint action of $\mathfrak{b}$. Since $Z^{0}$ is isomorphic to $G_{a}^{r}$ (in fact $r=3$ ), the analytic subgroup $A$ corresponding to $\mathfrak{a}$ is algebraic. The group $A$ is invariant by $\operatorname{ad}(H)$. Hence the subgroup $A H$ satisfies our requirement. 
Subcase (a-2). $R_{u}=\{e\}$.

Then the radical $R$ is a torus $G_{m}^{r}$ and $G$ is reductive. Then the radical $R$ coincides with the center of $G$. From our assumption (3.9), $G=R H$ $=\boldsymbol{G}_{m}^{r} H$. It follows from our hypothesis (3.1) $r=3$. An algebraic subgroup $K=G_{m} H$ satisfies the condition of corollary 2.8. Now it remains to examine

Case (b). The group $G$ is semi-simple.

Let $\alpha: \tilde{G} \rightarrow G$ be the universal covering of $G$. Then it is known that $\tilde{G}$ and $\alpha$ are algebraic (théorème 10, Chap. 8, Serre [7]). In fact $\alpha$ is finite. We identify by $\alpha$ the Lie algebras of $\tilde{G}$ and $G$. Let $\tilde{H}$ be the inverse image $\alpha^{-1}(H)$. Then evidently the variety $\tilde{G} / \tilde{H}$ is isomorphic to $G / H$. It is sufficient to construct an algebraic subgroup $\tilde{K}^{\prime} \subset \tilde{G}$ such that $\tilde{H} \subseteq \tilde{K}^{\prime}$ $\subsetneq \tilde{G}, \operatorname{dim} \tilde{H}<\operatorname{dim} \tilde{K}^{\prime}$. For, the image $\alpha\left(\tilde{K}^{\prime}\right)$ satisfy the condition of corollary 2.8 since $\alpha$ is finite. The operation $(\tilde{G}, \tilde{G} / \tilde{H})$ does not satisfy the condition (3.1) but the kernel of the homomorphism $\tilde{G} \rightarrow \operatorname{Aut}(\tilde{G} / \tilde{H})$ is the kernel of $\alpha$ which is finite. Let $\tilde{N}$ be a connected normal subgroup of positive dimension of $\tilde{G}$. Then by the same argument as at the biginning of the proof of the theorem, we may assume

$(3.9)^{\prime} \tilde{G}=\tilde{N} \tilde{H}$; the group $\tilde{N}$ acts transitively on $\tilde{G} / \tilde{N}$.

By proposition 1.8, the imprimitivity of the operation $(G, G / H)$ shows that the Lie subalgebra $\mathfrak{h} \subset g$ of $H$ (resp. $\tilde{H}$ ) in $G$ (resp. $\tilde{G}$ ) is not maximal. Let $\mathfrak{i}$ be a Lie subalgebra of $g$ such that $\mathfrak{h} \subseteq \subseteq \mathrm{g}$. Since the dimension of $G / H$ is 3 , the dimension of the vector space $g / f$ is 1 or 2 .

Subcase (b-1). There exists a Lie subalgebra $\mathfrak{f} \subset \mathfrak{g}$ such that $\mathfrak{h} \subset \mathfrak{l} \subset$ $\mathfrak{g}, \operatorname{dim} \mathfrak{g} / \mathfrak{t}=1$.

Lемма 3.10. The Lie subalgebra $\mathfrak{l}^{\prime}$ is algebraic; there exists a connected algebraic subgroup $\tilde{K} \subset \tilde{G}$ whose Lie algebra is $\mathfrak{k}$.

Proof of the lemma. It follows from proposition 1.8 a law chunk $\left(G^{\prime}, X^{\prime}\right)$ of analytic operation and a morphism of law chunks of analytic operation $(\varphi, f):(\tilde{G}, \tilde{G} / \tilde{N}) \rightarrow\left(G^{\prime}, X^{\prime}\right)$ such that the dimension of $X^{\prime}=1$ and the image $f$ has positive dimension. By Lie [6], any one dimensional law chunk of analytic operation is considered as a sub-law chunk of the analytic operation $\left(P G L_{2}, \boldsymbol{P}_{1}\right)$. Hence, we may assume $\left(G^{\prime}, X^{\prime}\right)=\left(P G L_{2}, \boldsymbol{P}_{1}\right)=\left(P G L_{2}\right.$, $P G L_{2} / B$ ) where $B$ is a Borel subgroup of $P G L_{2}$. The subgroup $B$ is alge- 
braic. Further, since $(\tilde{G}, \tilde{G} / \tilde{H})$ and $\left(P G L_{2}, P_{1}\right)$ are homogeneous spaces, we may assume that $f$ is defined at the coset $\tilde{H} \in \tilde{G} / \tilde{H}$ and the image $f(\tilde{H})$ is the coset $B \in P G L_{2} / B$. Since $\tilde{G}$ is simply connected, the local homomorphism $\varphi$ can be extended to a homomorphism of analytic groups, which is also denoted by $\varphi$. Set $\varphi_{*} ; \mathfrak{g} \rightarrow \mathfrak{S l}_{2}$ denote the Lie algebra homomorphism induced by $\varphi$ and $\mathfrak{b}$ be the Lie algebra of $B$. Then the inverse image $\varphi_{*}^{-1} \mathfrak{b}(=\mathfrak{f})$ is the Lie algebra of the inverse image $\varphi^{-1}(B)$ which is algebraic, since $\varphi$ is algebraic by théorème 10, Chap. 8, Serre [9]. q.e.d.

Remark 3.11. Since $\mathfrak{g}$ is semi-simple, $\varphi_{*}: \mathfrak{g} \rightarrow \mathfrak{g l}_{2}$ is surjective, the inclusion $\mathfrak{h} \subset \mathfrak{i m p l i e s} H^{0} \subset K$.

Let $\tilde{N}$ be the connected component of the kernel of $\varphi$. Since $\operatorname{dim} \tilde{G}$ $\geq 4>\operatorname{dim} \xi_{2}$, the dimension of $\tilde{N}$ is positive. The $\tilde{N}$-orbits on $\tilde{G} / \tilde{H}$ are contained in the fibres of the canonical morphism $\tilde{G} / \tilde{H}^{0} \rightarrow \tilde{G} / \tilde{K}=\boldsymbol{P}_{1}$ hence they are at most of dimension $\leq 2$. On the other hand the $\tilde{N}$-orbits on $\tilde{G} / \tilde{H}$ are the images of the $\tilde{N}$ orbits on $\tilde{G} / \tilde{H}^{0}$ by the canonical morphism $\tilde{G} / \tilde{H}^{0} \rightarrow \tilde{G} / \tilde{H}$. Since the morphism $\tilde{G} / \tilde{H}^{0} \rightarrow \tilde{G} / \tilde{H}$ is finite, the $\tilde{N}$-orbits on $\tilde{G} / \tilde{H}$ are at most of dimension 2. Hence the operation of $\tilde{N}$ on $\tilde{G} / \tilde{H}$ is not transitive. Subcase (b-1) never happens under the hypotheses (3.9)'.

Subcase (b-2). There does not exist any Lie subalgebra $\mathfrak{f}$ such that $\mathfrak{h} \sqsubseteq \mathfrak{f} g \mathfrak{g}, \operatorname{dim} \mathfrak{g} / \mathfrak{t}=1$. But there exists a Lie subalgebra $\mathfrak{l}$ such that $\mathfrak{h} \subsetneq \mathfrak{l} \subsetneq \mathfrak{g}, \operatorname{dim} \mathfrak{g} / \mathfrak{l}=2$.

This condition shows by proposition 1.8 that there exists a primitive law chunk of analytic of operation $\left(G^{\prime}, X^{\prime}\right)$ and a morphism of law chunks of analytic operations $(\varphi, f):\left(\tilde{G}^{a n}, \tilde{G} / \tilde{H}^{a n}\right) \rightarrow\left(G^{\prime}, X^{\prime}\right)$ such that $\varphi$ induces a surjective morphism of Lie algebras of $\tilde{G}$ and $G^{\prime}$. By Lie [6], we know all the primitive operation of dimension 2. Namely, the law chunk of analytic operation $\left(G^{\prime}, X^{\prime}\right)$ is (locally isomorphic to) one of the following:

(i) The full projective group $\left(P G L_{3}, \boldsymbol{P}_{2}\right)$

(ii) The affine transformation group $\left(T A_{2}, A_{2}\right)$

(iii) The special affine transformation group $\left(S T A_{2}, A_{2}\right)$.

(affine transformations with determinant $=1$ ).

This shows, in particular the law chunks of analytic operation $\left(G^{\prime}, X^{\prime}\right)$ can be extended to global analytic operations. Notice also the analytic operations (i), (ii), (iii) are algebraic.

Lemma 3.12. The law chunk of analytic operation $\left(G^{\prime}, X^{\prime}\right)$ is locally isomorphic to $\left(P G L_{3}, \boldsymbol{P}_{2}\right)$. 
Proof of the lemma. Since the morphism of analytic group germs $\varphi$ induces a surjective morphism of Lie algebras and the Lie group $\tilde{G}$ is semi-simple, the Lie group $G^{\prime}$ is semi-simple. The Lie groups $T A_{2}$ and $S T A_{2}$ are not semi-simple. Hence the law chunk of analytic operation $\left(G^{\prime}, X^{\prime}\right)$ is locally isomorphic to $\left(P G L_{3}, P_{2}\right)$.

q.e.d.

Now we have a morphism of law chunks of analytic operation $(\varphi, f)$ : $\left(\tilde{G}^{a n}, \tilde{G} / \tilde{H}^{a n}\right) \rightarrow\left(P G L_{3}, \boldsymbol{P}_{2}\right)$. Since $\tilde{G}$ is a simply connected, the morphism $\varphi$ is not only a morphism of analytic group germs but also a morphism of analytic groups. Let $P$ be a parabolic subgroup of $P G L_{3}$ consisting of matrices $\left(\begin{array}{lll}* & * & * \\ 0 & * & * \\ 0 & * & *\end{array}\right) \in P G L_{3}$ hence $\left(P G L_{3}, P_{2}\right) \simeq\left(P G L_{3}, P G L_{3} / P\right)$. Since we are dealing with homogeneous spaces, we may assume that the local morphism $f$ is defined at the coset $\tilde{H} \in \tilde{G} / \tilde{H}$ and the image $f(\tilde{H})$ is the coset $P \in \boldsymbol{P}_{2}$. Let $z$ be the Lie subalgebra of $P$ and $\varphi_{*}: \mathfrak{g} \rightarrow \mathfrak{Z Y}_{3}$ be the homomorphism of Lie algebras induced by $\varphi$. The Lie subalgebra $\mathfrak{l}$ of $\mathfrak{g}$ coincides with $\varphi_{*}^{-1}(\precsim)$. By théorème 10, Chap. 8, Serre [9], the morphism $\varphi$ is algebraic. Hence the algebraic subgroup $\varphi^{-1}(P)$ of $\tilde{G}$ corresponds to the Lie subalgebra $\mathfrak{l}=\varphi_{*}^{-1}(\mathfrak{z})$. Putting $\tilde{L}=\varphi^{-1}(P)$, we have $\tilde{H}^{0} \subset \tilde{L}$ because there is an inclusion of Lie subalgebras $\mathfrak{h} \subset \mathfrak{l}$. Now let $f^{\prime}$ be the canonical projection $\tilde{G} / \tilde{H}^{0} \rightarrow \tilde{G} / \tilde{L}=\tilde{G} / \varphi^{-1}(P) \simeq P G L_{3} / P \simeq \boldsymbol{P}_{2}$. The map $f^{\prime}$ is a morphism of algebraic variety $\tilde{G} / \tilde{H}^{0}$ onto $\boldsymbol{P}_{2}$. Then, we have a morphism of algebraic operations $\left(\varphi, f^{\prime}\right):\left(\tilde{G}, \tilde{G} / \tilde{H}^{0}\right) \rightarrow\left(P G L_{3}, \boldsymbol{P}_{2}\right)$. There is a canonical morphism of algebraic operations $(\mathrm{Id}, g):\left(\tilde{G}, \tilde{G} / \tilde{H}^{0}\right) \rightarrow(\tilde{G}, \tilde{G} / \tilde{H})$ induced by the inclusion $\tilde{H}^{0} \subset \tilde{H}$. The morphism $g$ of algebraic varieties is finite since $\tilde{H}$ is algebraic.

We have a following diagram;

$$
\begin{aligned}
& \left(\tilde{G}, \tilde{G} / \tilde{H}^{0}\right) \stackrel{(\varphi, f)}{\longrightarrow}\left(P G L_{3}, P_{2}\right) \\
& (\tilde{G}, g) \\
& (\tilde{G}, \tilde{G} / \tilde{H})
\end{aligned}
$$

The morphism $(\varphi, f)$ can not be factorized by $(\varphi, g)$ in the category of the algebraic operations but we can complete the diagram above to a commutative diagram locally and analytically:

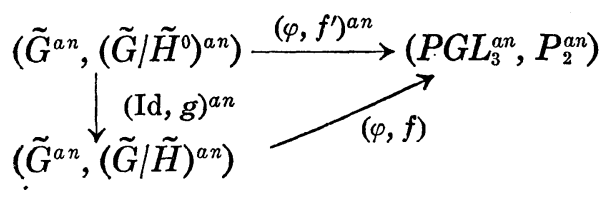


As the morphism $\varphi$ is surjective, $\operatorname{dim} \tilde{G} \geq \operatorname{dim} P G L_{3}=8$. Let us first study the case $\operatorname{dim} \tilde{G}>8$. Then the kernel of $\varphi$ has positive dimension. Let $\tilde{N} \neq\{e\}$ denote the connected component of the kernel of $\varphi$. Then $\tilde{N}$ leaves fixed all the fibres of $f^{\prime}$. Hence the connected normal subgroup $\tilde{N}$ of positive dimension does not act transitively. Since the projection $g: \tilde{G} / \tilde{H}^{0}$ $\rightarrow \tilde{G} / \tilde{H}$ is finite, $\tilde{N}$ does not act transitively on $\tilde{G} / \tilde{H}$ which contradicts our hypothesis $(3.9)^{\prime}$. This subcase with $\operatorname{dim} \tilde{G}>8$ can not occur under the hypotheses $(3.9)^{\prime}$. It remains to treat the case $\operatorname{dim} \tilde{G}=8$. Then, the Lie algebra $g$ is isomorphic to $\mathfrak{g l}_{3}$ hence $\tilde{G}=S L_{3}$. We may assume that the morphism $\varphi: S L_{3}=\tilde{G} \rightarrow P G L_{3}=S L_{3} /$ center of $S L_{3}$ is the canonical projection. The Lie subalgebra $\mathfrak{l}$ consists of the matrices $\left(\begin{array}{lll}* & * & * \\ 0 & * & * \\ 0 & * & *\end{array}\right)$. The Lie algebra $\mathfrak{h}$ is a subalgebra of $\mathfrak{l}$.

LEMma 3.13. Assume $\tilde{G}=S L_{3}$. Then one of the following assertions is true.

(1) The Lie subalgebra $\mathfrak{h}$ is parabolic in $\mathrm{g}$.

(2) The Lie subalgebra $\mathfrak{h}$ coincides with $\left\{\left(\begin{array}{lll}0 & * & * \\ 0 & * & * \\ 0 & * & *\end{array}\right) \in \mathfrak{l}\right\}$,

Proof of the lemma. Putting $\mathfrak{n}=\left\{\left(\begin{array}{lll}0 & * & * \\ 0 & 0 & 0 \\ 0 & 0 & 0\end{array}\right) \in \mathfrak{l}\right\}, \mathfrak{g}^{\prime}=\left\{\left(\begin{array}{lll}* & 0 & 0 \\ 0 & * & * \\ 0 & * & *\end{array}\right) \in \mathfrak{l}\right\}$, we get an exact sequence;

$$
0 \longrightarrow \mathfrak{n} \stackrel{\alpha}{\longrightarrow} \mathfrak{r} \stackrel{\beta}{\longrightarrow} \mathfrak{g}^{\prime} \longrightarrow 0 \text {. }
$$

Since $\operatorname{dim} \mathfrak{n}=2, \operatorname{dim} \mathfrak{h}=5, \operatorname{dim} \mathfrak{l}=6$, we have either

(i) The Lie algebra $\mathfrak{h} \cap \mathfrak{n}$ is one dimensional or

(ii) $\mathfrak{n} \subset \mathfrak{h}$.

Let us show the first case does not occur. If $\operatorname{dim} \mathfrak{h} \cap \mathfrak{n}=1$, then $\beta(\mathfrak{h})=\mathfrak{g}$. The Lie algebra $\mathfrak{h} \cap \mathfrak{n}$ is invariant by $\mathfrak{g}^{\prime}$. But this is impossible because $\mathfrak{n}$ is an irreducible $\mathfrak{g}$-module. If $\mathfrak{n} \subset \mathfrak{h}$, the image $\beta(\mathfrak{h})$ is a Lie subalgebra of dimension 3 of $\mathfrak{g}^{\prime}$. Hence the Lie algebra $\beta(\mathfrak{h})$ is one of the following; $\left\{\left(\begin{array}{lll}0 & 0 & 0 \\ 0 & * & * \\ 0 & * & *\end{array}\right) \in \mathfrak{g}^{\prime}\right\},\left\{\left(\begin{array}{ccc}* & 0 & 0 \\ 0 & a \\ 0 & a\end{array}\right) \in \mathfrak{g}^{\prime}\right\}$ where $\mathfrak{a}$ is a Borel subalgebra of $\mathfrak{g l}_{2}$. It follows from this that the Lie algebra $\mathfrak{h}$ coincides with one of the following; $\left\{\left(\begin{array}{lll}0 & * & * \\ 0 & * & * \\ 0 & * & *\end{array}\right) \in \mathfrak{S I}_{3}\right\}, \quad\left\{\left(\begin{array}{ccc}* & * & * \\ 0 & a \\ 0 & a\end{array}\right) \in \mathfrak{B I}_{3}\right\}$. The following lemma completes the proof of the theorem. 
LEMMA 3.15. If $\operatorname{dim} \tilde{G}=S L_{3}$, then the algebraic subgroup $\tilde{H} \subset S L_{3}$ is contained in the parabolic subgroup $\tilde{L}=\left\{\left(\begin{array}{lll}* & * & * \\ 0 & * & * \\ 0 & * & *\end{array}\right) \in S L_{3}\right\}$.

Proof of the lemma. By the inclusion of Lie subalgebras $\mathfrak{h} \subset \mathfrak{l}$, we have $\tilde{H}^{0} \subset \tilde{L}$. If we are in the first case of Lemma 3.13 , then it is well known that a parabolic subgroup is connected hence $\tilde{H}=\tilde{H}^{0} \subset \tilde{L}$. In the second case of lemma $3.13, \tilde{H}^{0}=\left\{\left(\begin{array}{lll}1 & * & * \\ 0 & * & * \\ 0 & * & *\end{array}\right) \in S L_{3}\right\}$. Let us assume $\tilde{H} \varnothing \tilde{L}$. Then there exists an element $\left(\begin{array}{cc}a & v \\ w & A\end{array}\right) \in S L_{3}$ of $\tilde{H}, a \in C, v,{ }^{t} w \in C^{2} A \in G L_{2}$ with $w \neq 0$. For any $B \in S L_{2}$, a matrix $\left(\begin{array}{ccc}1 & 0 & 0 \\ 0 & B \\ 0 & B\end{array}\right)$ belongs to $\tilde{H}$ hence the product $\left(\begin{array}{ll}1 & \\ & B\end{array}\right)\left(\begin{array}{cc}a & v \\ w & A\end{array}\right)=\left(\begin{array}{cc}a & v \\ B w & B A\end{array}\right) \in \tilde{H}$ varing $B \in S L_{2}, \quad B w$ can be any non-zero vector $C^{2}$. Consider the canonical map

$$
\varphi: \tilde{H} / \tilde{H}^{0} \rightarrow \tilde{H} / \tilde{H} \cap \tilde{L} \subset S L_{3} / \tilde{L}=\boldsymbol{P}_{2} ; \varphi\left\{\left(\begin{array}{lll}
x_{11} & x_{12} & x_{13} \\
x_{21} & x_{22} & x_{23} \\
x_{31} & x_{32} & x_{33}
\end{array}\right)\right\}=\left(\begin{array}{l}
x_{11} \\
x_{21} \\
x_{31}
\end{array}\right) \in \boldsymbol{P}_{2} .
$$

The calculation above shows that the image $\varphi\left(\tilde{H} / \tilde{H}^{0}\right)$ is not finite which is a contradiction since $\tilde{H}$ is an algebraic group.

Corollary 3.16.1. Let $(G, G / H)$ be an algebraic homogeneous space satisfying the condition (3.1) such that $G / H$ is rational and of dimension 3. If $\operatorname{dim} G \geq 4$ and $(G, G / H)$ is imprimitive, then there is a closed (algebraic) subgroup $K \subsetneq G$ such that $\operatorname{dim} H<\operatorname{dim} K$ and $K / H$ is irreducible.

Proof. We have only to check the connectedness of $K / H$. Let us examine the proof of the theorem. If $G$ is solvable, then we have a connected normal $N$ subgroup of dimension 1 . Then it is sufficient to put $K=H N$. The variety $K / H=H N / H=N / N \cap H$ is irreducible. In case (a), we have shown that there exists a connected normal subgroup $N$ such that $K=H N$. Hence for the same reason as above, the variety $K / H$ is irreducible. In case (b), we work with $\tilde{G}$ rather than $G$. In this case it is sufficient to check in $\tilde{G}$. In the cases $(b-1)$ and $(b-2)$ with $\operatorname{dim} \tilde{G}>8$, we found a connected normal subgroup as above. In the case (b-2) with $\tilde{G}=S L_{3}$ we have shown that $\tilde{H}$ is contained in the connected subgroup $\tilde{L}$. Hence the variety $\tilde{L} / \tilde{H}$ is irreducible. 
In the proof of the theorem, when we studied case (a-1) and (a-2), we $\operatorname{did}$ not use the hypothesis that $\operatorname{dim} X=3, \operatorname{dim} G \geq 4$. Hence we have,

CoRollary 3.16.2. Let $G$ be a connected algebraic group, $X$ a rational variety and $(G, X)$ an algebraic operation satisfying the condition (3.1). If $(G, X)$ is imprimitive and $G$ is not semi-simple, then $(G, X)$ is of de Jonquières type.

We ask whether in $\mathrm{Cr}_{3}$ there is an imprimitive operation which is not of de Jonquières type.

By theorem 3.7, it is sufficient to study the imprimitive algebraic operations $(G, X)$ with $\operatorname{dim} \mathrm{G} \leq 3$. If $G$ is solvable, the algebraic operation $(G, X)$ is of de Jonquières type by corollary 2.6. Since an algebraic group of dimension $\leq 2$ is solvable, the algebraic operation $(G, X)$ is of de Jonquières type if $\operatorname{dim} G \leq 2$. Thus we have to examine the operations $(G, X)$ such that $G$ is semi-simple and of dimension 3, i.e. $G$ is isomorphic to $\mathrm{SL}_{2}$ or $\mathrm{SO}_{3}$. Moreover by corollary 2.5, $(G, X)$ is of de Jonquières type if $(G, X)$ is not generically transitive. Therefore it remains to study only the homogeneous spaces $(G, G / H)$ where $G$ is $S L_{2}$ or $S O_{3}$. Since the dimension of $G$ and $G / H$ coincide, $H$ is a finite subgroup of $G$. For any finite subgroup $H$ of $G$ ( $\left.=S L_{2}, S O_{3}\right),\left(G^{a n}, G / H^{a n}\right)$ is isomorphic to $\left(S L_{2}^{a n}\right.$, $\left.S L_{2}^{a n}\right)$ as a law chunk of analytic operation. Therefore by proposition 1.7, $(G, G / H)$ is imprimitive. We know all the finite subgroups in $G$. Since there is a morphism of degree 2 of $\mathrm{SL}_{2}$ onto $\mathrm{SO}_{3}$, all the finite subgroups of $\mathrm{SO}_{3}$ are the images of finite subgroups of $\mathrm{SL}_{2}$.

Proposition 3.17 (Blichfeldt [1]). A finite subgroup $\Gamma$ of $S L_{2}$ is conjugate to one of the following;

(A) Cyclic group.

$$
\left\{\left(\begin{array}{cc}
\varepsilon^{m} & 0 \\
0 & \varepsilon^{-m}
\end{array}\right) \mid \varepsilon=e^{2 \pi i / N}, m=0,1, \cdots, N-1\right\}, N=1,2,3, \cdots .
$$

(B) Dihedral group. A subgroup generated by

$$
\left(\begin{array}{cc} 
\pm \varepsilon^{m} & 0 \\
0 & \pm \varepsilon^{-m}
\end{array}\right), \quad\left(\begin{array}{rr}
0 & 1 \\
-1 & 0
\end{array}\right), \quad \varepsilon=e^{2 \pi i / N} \quad \text { where } \quad N=1,2,3, \cdots
$$

(C) Tetrahedral group. A subgroup generated by

$$
\left(\begin{array}{cc}
(-1+i) / 2 & (-1+i) / 2 \\
(1+i) / 2 & (-1-i) / 2
\end{array}\right), \quad\left(\begin{array}{cc}
i & 0 \\
0 & -i
\end{array}\right) .
$$


(D) Octahedral group. A subgroup generated by

$$
\left(\begin{array}{cc}
(-1+i) / 2 & (-1+i) / 2 \\
(1+i) / 2 & (-1-i) / 2
\end{array}\right), \quad\left(\begin{array}{cc}
(1+i) / \sqrt{2} & 0 \\
0 & (1-i) / \sqrt{2}
\end{array}\right) .
$$

(E) Icosahedral group. A subgroup generated by the tetrahedral group and

$$
\left(\begin{array}{cc}
i / 2 & \beta-i \gamma \\
-\beta-i \gamma & -1 / 2
\end{array}\right) \quad \text { where } \beta=\frac{1-\sqrt{5}}{4}, \gamma=\frac{1+\sqrt{5}}{4}
$$

LEMMA 3.18. Let $\Gamma$ be a finite subgroup of the algebraic group $G$ which is isomorphic to $\mathrm{SL}_{2}$ or $\mathrm{SO}_{3}$. Then the quotient variety $\mathrm{G} / \Gamma$ is rational.

Proof. Let $B$ be a Borel subgroup of $G$. Then the quotient variety $B \backslash G$ is a projective rational curve hence $\boldsymbol{P}_{1}$. We have a following commutative diagram;

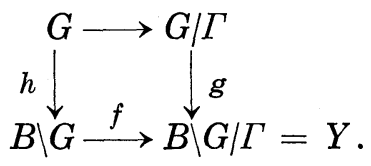

The morphism $h: G \rightarrow B \backslash G$ is a principal $B$-bundle. Since $\Gamma$ is finite, the set $F=\{x \in B \backslash G \mid x g=x$ for some $g \in \Gamma, g \neq e\}$ is closed. When $G=S L_{2}$, we may assume that the intersection of $\Gamma$ with the center $Z=\left\{ \pm I_{2}\right\}$ of $S L_{2}$ reduces to the unit element since if $\Gamma \supset Z$, considering the isogeny $\varphi: S L_{2} \rightarrow S L_{2} / Z \simeq S O_{3}$, we get $S L_{2} / \Gamma \simeq S O_{3} / \varphi(\Gamma)$. It follows from this assumption $\Gamma$ operates effectively on $B \backslash G=\boldsymbol{P}^{1}$ hence $F \subseteq B \backslash G=\boldsymbol{P}^{1}$. The group $\Gamma$ operates on $U=B \backslash G-F$ freely and $f$ is étale on $U$. Let $X=$ $h^{-1}(U)$, then the group $\Gamma$ operates freely on the principal $B$-bundle $h: X$ $\rightarrow U$. By the descent theory, $X / \Gamma \rightarrow U / \Gamma$ is a principal $B$-bundle and $X / \Gamma$ is an open subset of $G / \Gamma$. Since $B$ is solvable, the principal $B$-bundle $X \mid \Gamma \rightarrow U / \Gamma$ is locally trivial for the Zariski topology. Thus $X / \Gamma$ is birational to $B \times(U / \Gamma)$. Since $B=\boldsymbol{G}_{m} \cdot \boldsymbol{G}_{a}, B$ is rational and $(U / \Gamma)$ is rational by the Lüroth theorem. Therefore $X / \Gamma$ is rational hence $G / \Gamma$ is rational.

LEMma 3.19. Let $\Gamma$ be a finite subgroup of $S L_{2}$. An algebraic operation $\left(S L_{2}, S L_{2} / \Gamma\right)$ is of de Jonquières type if $\Gamma$ is conjugate to one of the finite subgroups (A), (B).

Proof. We may assume that $\Gamma$ is one of the finite subgroups (A), (B). If $\Gamma$ is cyclic, the cyclic groups $\Gamma$ is contained in $K=\left\{\left(\begin{array}{ll}a & 0 \\ 0 & a^{-1}\end{array}\right) \mid a \in C^{*}\right\}$. 
The natural projection $\left(S L_{2}, S L_{2} / \Gamma\right) \rightarrow\left(S L_{2}, S L_{2} / K\right)$ shows that $\left(S L_{2}, S L_{2} / \Gamma\right)$ is of de Jonquières type. If $\Gamma$ is dihedral, $\Gamma$ is contained in a algebraic subgroup generated by $\left(\begin{array}{ll}a & 0 \\ 0 & a^{-1}\end{array}\right),\left(\begin{array}{rr}0 & 1 \\ -1 & 0\end{array}\right), a \in C^{*}$. The same argument shows that $\left(S L_{2}, S L_{2} / \Gamma\right)$ is of de Jonquières type.

LEMMA 3.20. Let $\Gamma$ be a finite subgroup of $S L_{2}$. An algebraic operation $\left(S L_{2}, S L_{2} / \Gamma\right)$ is not of de Jonquières type if $\Gamma$ is conjugate to one of the finite subgroups (C), (D), (E).

Proof. We may assume that $\Gamma$ is one of the finite subgroups (C), (D), (E). It is sufficient to show that there is no proper algebraic subgroup $K$ of positive dimension containing $\Gamma$, by corollary 2.8. Suppose that there exists such a $K$. Let $\mathfrak{l}$ be the Lie algebra of $K$. The Lie subalgebra $\mathfrak{f}$ is conjugate to $\left\{\left(\begin{array}{rr}a & 0 \\ 0 & -a\end{array}\right) \mid a \in C\right\},\left\{\left(\begin{array}{ll}0 & b \\ 0 & 0\end{array}\right) \mid b \in C\right\}$ or $\left\{\left(\begin{array}{rr}a & b \\ 0 & -a\end{array}\right) \mid a, b \in C\right\}$.

(3.21) Assume that $\operatorname{dim} \mathfrak{f}=2$.

Then, $\mathfrak{f}$ is conjugate to $\left\{\left(\begin{array}{rr}a & b \\ 0 & -a\end{array}\right) \mid a, b \in C\right\}$. Then $K$ is a Borel subgroup. As the algebraic subgroup $K$ is conjugate to $\left\{\left(\begin{array}{ll}a & b \\ 0 & a^{-1}\end{array}\right) \mid a \in C^{*}, b \in C\right\}, \Gamma$ is conjugate to a subgroup of triangular matrices. This shows that the inclusion $\Gamma \subset S L_{2}$ is not an irreducible representation. This is a contradiction. Since $\Gamma$ is not contained in any two dimensional subgroup, $\Gamma$ is contained in the normalizer of a one-dimensional subgroup.

(3.22) Assume $\mathfrak{l}$ is conjugate to $\left\{\left(\begin{array}{ll}0 & b \\ 0 & 0\end{array}\right) \mid b \in C\right\}$.

The normalizer of the Lie subalgebra $\left\{\left(\begin{array}{ll}0 & b \\ 0 & 0\end{array}\right) \mid b \in C\right\}$ is a Borel subalgebra consisting of all the upper triangular matrices of $\mathcal{B l}_{2}$. Thus, $\Gamma$ is contained in a Borel subgroup. This is impossible as we have shown in (3.21).

(3.23) Assume $\mathfrak{E}$ is conjugate to $\left\{\left(\begin{array}{rr}a & 0 \\ 0 & -a\end{array}\right) \mid a \in C\right\}$. The connected component $K^{0}$ is conjugate to $T=\left\{\left(\begin{array}{cc}a & 0 \\ 0 & a^{-1}\end{array}\right) \mid a \in C^{*}\right\}$. The normalizer $N$ of $T$ is a subgroup generated by $T$ and $\left(\begin{array}{rr}0 & 1 \\ -1 & 0\end{array}\right)$.

We have an exact sequence;

$$
1 \longrightarrow T \longrightarrow N \longrightarrow Z / 2 Z \longrightarrow 0 \text {. }
$$

Since the group $\Gamma$ is a subgroup of $N$ and $\Gamma$ is not cyclic, there exist a 
finite cyclic group $A$ and an exact sequence;

$$
1 \longrightarrow A \longrightarrow \Gamma \longrightarrow Z / 2 Z \longrightarrow 0 \text {. }
$$

If we put $Z=\left\{ \pm I_{2}\right\}, Z<\Gamma$ and $\Gamma / Z$ is isomorphic to $\mathfrak{A}_{4}, \mathfrak{S}_{4}$ or $\mathfrak{A}_{5}$ according as $\Gamma$ is tetrahedral, octahedral or icosahedral. (Blichfeldt [1]). Since these groups are not cyclic, we have an exact sequence

$$
1 \longrightarrow B \longrightarrow \Gamma / Z \longrightarrow Z / 2 Z \longrightarrow 0
$$

where $B$ is a finite cyclic group. But the permutation groups $\mathfrak{U}_{4}, \mathfrak{S}_{4}, \mathfrak{U}_{5}$ can not be an extension of $Z / 2 Z$ by a cyclic group.

LEMMA 3.24. Let $\Gamma$ be a finite subgroup of $S L_{2}$. Let $\pi: S L_{2} \rightarrow S O_{3}$ be a covering morphism of degree 2. Then, an algebraic operation $\left(S L_{2}, S L_{2} / \Gamma\right)$ is of de Jonquières type if and only if $\left(\mathrm{SO}_{3}, \mathrm{SO}_{3} / \pi(\Gamma)\right)$ is of de Jonquières type.

Proof. It is sufficient by corollary 2.8 to consider the image and the inverse image of algebraic subgroups containing $\Gamma$ or $\pi(\Gamma)$.

(3.25) Here is a complete list of imprimitive algebraic operations which are not of de Jonquières type;

$\left(S L_{2}, S L_{2} / \Gamma\right),\left(S_{3}, S O_{3} / \pi(\Gamma)\right)$ where $\Gamma$ is the tetrahedral octahedral or icosahedral subgroup of $S L_{2}$.

Among the algebraic operations in (3.25), only $\left(\mathrm{SO}_{3}, \mathrm{SO}_{3} / \pi(\Gamma)\right)$ satisfies the condition (3.1). Therefore,

THEOREM 3.26. Every imprimitive connected algebraic group $G$ in $\mathrm{Cr}_{3}$ is conjugate to one of the following algegraic operations.

(A) de Jonquières type operations

(B) $\left(\mathrm{SO}_{3}, \mathrm{SO}_{3} / \Gamma\right)$ where $\Gamma$ is the tetrahedral, the octahedral or the icosahedral subgroup of $\mathrm{SO}_{3}$.

LEMMA 3.27. The algebraic operation $\left(\mathrm{SO}_{3}, \mathrm{SO}_{3} / \pi(\Gamma)\right)$ is not contained in a primitive algebraic operation of $\mathrm{Cr}_{3}$ if $\Gamma$ is either octahedral or icosahedral.

Proof. Every primitive algebraic operation is contained in $\left(P G L_{4}, \boldsymbol{P}_{3}\right)$ or $\left(P G L_{5}\right.$, quadric $\left.\subset P_{4}\right)$ by [10]. Suppose that $\left(S O_{3}, S O_{3} / \pi(\Gamma)\right)$ is an algebraic suboperation of $\left(P G L_{4}, P_{3}\right)$. The algebraic operation $\left(P G L_{4}, P_{3}\right)$ are linearized; this is the projectification of a linear representations of $S L_{4}$. Therefore, there is a linear representation $\rho: S L_{2} \rightarrow S L_{4}=S L(E)$ and a linear sub- 
space $L \subset E$ of dimension 1 such that, for $g \in S L_{2}, g$ belongs to $\Gamma$ if and only if $\rho(g)(L) \subset L$. In particular if $g \in \Gamma$, then $\rho(g) L \subset L$. The $S L_{2}$-module $E$ is the direct sum of irreducible $S L_{2}$-modules of dimension $\leq 4$, i.e. vector spaces of the homogeneous polynomials of 2 variables of degree $\leq 3$. But the group $\Gamma$ has no semi-invariant of degree $\leq 3$ (neunter Abschnitt, Weber [11]). Hence, the operation $\left(\mathrm{SO}_{3}, \mathrm{SO}_{3} / \pi(\Gamma)\right)$ is not an algebraic suboperation of $\left(\mathrm{PGL}_{4}, \mathrm{P}_{3}\right)$. Assume now that $\left(\mathrm{SO}_{3}, \mathrm{SO}_{3} / \Gamma\right)$ is an algebraic suboperation of $\left(\mathrm{PSO}_{5}\right.$, quadric $\left.\subset \mathrm{P}_{4}\right)$. It follows that $\left(\mathrm{SO}_{3}, \mathrm{SO}_{3} / \Gamma\right)$ is an algebraic suboperation of $\left(P G L_{5}, P_{5}\right)$. The same argument as above shows that $\Gamma$ has a semi-invariant of degree $\leq 4$. This is impossible for the same reason as above.

Proposition 3.28. Let $\Gamma$ be the tetrahedral subgroup of $S L_{2}$. Then the algebraic operation $\left(\mathrm{SO}_{3}, \mathrm{SO}_{3} / \pi(\Gamma)\right)$ is an algebraic suboperation of $\left(\mathrm{SO}_{5}\right.$, quadric $\subset P_{4}$ ).

Proof. Let $f=a_{0} x_{1}^{4}+4 a_{1} x_{1}^{3} x_{2}+6 a_{2} x_{1}^{2} x_{2}^{2}+4 a_{3} x_{1} x_{2}^{3}+a_{4} x_{2}^{4}$.

Then, $i=2\left(a_{0} a_{4}-4 a_{1} a_{3}+3 a_{2}^{2}\right)$ is invariant if we make a substitution

$$
\left(\begin{array}{l}
x_{1} \\
x_{2}
\end{array}\right) \longmapsto A\left(\begin{array}{l}
x_{1} \\
x_{2}
\end{array}\right), \quad A \in S L_{2},
$$

p. 135, Clebsch [3]. By p. 275, Weber [11], $\Phi_{1}=x_{1}^{4}+2 \sqrt{-3} x_{1}^{3} x_{2}^{2}+x_{2}^{4}$ is invariant by $\Gamma$. Let us work on the projective space $P_{4} \cong P(E)$ where $E$ is the vector space of homogeneous polynomials in $x_{1}, x_{2}$ of degree 4 . Then $S L_{2}$ operates on $E$ hence on $P(E)$. Since there is no proper algebraic subgroup of positive dimension containing $\Gamma$ and since the tetrahedral group and icosahedral group have no semi-invariant of degree $\leq 4$, the $S L_{2}$-orbit of $\Phi_{1} \in P(E)$ is isomorphic to $S L_{2} / \Gamma$. We shall determine the defining equation of the closure $\bar{X}$ in $P(E)$. Since $\bar{X}$ is of dimension 3 , $\bar{X}$ is defined by a homogeneous polynomial in $a_{0}, a_{1}, \cdots, a_{6}$. Since for the polynomial $\Phi_{1}, i=0$ and $i$ is invariant, $i$ is a homogeneous polynomial in $a_{0}, a_{1}, \cdots, a_{6}$ vanishing on $\bar{X}$. Hence, $\bar{X}$ is a non-singular quadric in $\boldsymbol{P}_{4}$. Therefore $\left(\mathrm{SO}_{3}, \mathrm{SO}_{3} / \pi(\Gamma)\right)$ is an algebraic suboperation of $\left(\mathrm{SO}_{5}\right.$, quadric $\left.\subset \boldsymbol{P}_{4}\right)$

By summing up what we have done, we get

Theorem 3.29. A connected algebraic subgroup of the Cremona group $\mathrm{Cr}_{3}$ of three variables is conjugate to one of the following algebraic operations.

(1) Primitive operations

$$
\left(\mathrm{PGL}_{4}, \boldsymbol{P}_{3}\right),\left(\mathrm{PSO}_{5}, \text { quadric } \subset \boldsymbol{P}_{4}\right) \text {. }
$$




\section{(2) Imprimitive operations}

(A) de Jonquières type operations

(B) $\left(\mathrm{SO}_{3}, \mathrm{SO}_{3} / \Gamma\right)$ where $\Gamma$ is octahedral or icosahedral subgroup of $\mathrm{SO}_{3}$.

There is no inclusion among the operations in (1) and (2-B).

\section{REFERENCES}

[1] Blichfeldt, H. F., Finite collineation groups, The university of Chicago press, Chicago 1917.

[2] Bourbaki, N., Groupes et algèbres de Lie, Chapitres 2 et 3, Hermann, Paris 1972.

[ 3 ] Clebsch, A., Theorie der binären algebraischen Formen, Teubner, Leipzig 1872.

[4] Enriques, F. e Fano, G., Sui gruppi di transformazioni cremoniane dello spazio, Annali di Matématica pura ed applicata, s. $2^{\mathrm{a}}, 15$ (1897), 59-98.

[5] Fano, G., I gruppi di Jonquières generalizzati, Atti della. R. Acc. di Torino, $3^{\circ}$ (1898), 221-271.

[6] Lie, S., Theorie der Transformations gruppen, Teubner, Leipzig 1893.

[ 7 ] Matsumura, H., On algebraic groups of birational transformations, Lincei-R. Sc. fis. mat. e nat., 34 (1963), 151-155.

[ 8 ] Rosenlicht, M., Some basic theorems on algebraic groups, Amer. J. of Math., 78 (1956), 401-443.

[ 9 ] Serre, J.-P., Algèbres de Lie semi-simples complexes, Benjamin, New York 1966.

[10] Umemura, H., Sur les sous-groupes algébriques primitifs du groupe de Cremona à trois variables, Nagoya, Math., J., 79 (1980), 47-67.

[11] Weber, H., Lehrbuch der Algebra, Zweiter Band, Braunschweig 1899.

Department of Mathematics

Faculty of Science

Nagoya University

Chikusa-ku, Nagoya, 464, Japan 